\title{
Lifting The Burden: the first 7 years
}

\author{
Timothy J. Steiner • Gretchen L. Birbeck • \\ Rigmor Jensen · Zaza Katsarava · Paolo Martelletti • \\ Lars Jacob Stovner
}

Received: 20 October 2010/Accepted: 21 October 2010/Published online: 16 November 2010

(C) The Author(s) 2010. This article is published with open access at Springerlink.com

In September 2003, the World Health Organization (WHO) signed a Memorandum of Understanding that brought into being the Global Campaign against Headache (GC), known as Lifting The Burden.

This truly important event for people worldwide affected by headache signalled WHO's recognition of headache disorders as a global public-health priority. It did not come about easily: there are many competing claims upon WHO's limited resources, and WHO accords priority only where it is manifestly due. Headache disorders are highly prevalent, ubiquitous, often lifelong and disabling. These burdens persist despite that headache disorders are to a

T. J. Steiner $(\bowtie) \cdot$ L. J. Stovner

Department of Clinical Neuroscience,

Norwegian University of Science and Technology (NTNU),

7006 Trondheim, Norway

e-mail: t.steiner@imperial.ac.uk

T. J. Steiner

Imperial College London, London, UK

G. L. Birbeck

Michigan State University, East Lansing, MI, USA

G. L. Birbeck

Chikankata Health Services, Mazabuka, Zambia

R. Jensen

Danish Headache Centre, Glostrup, Denmark

R. Jensen

University of Copenhagen, Copenhagen, Denmark

Z. Katsarava

University of Essen, Essen, Germany

P. Martelletti

Sapienza University of Rome, Rome, Italy large extent treatable. Headache disorders therefore fulfil all of the criteria against which WHO assesses priority. We all knew this, but WHO required proof of it. This was quite right, and proof was provided, first at a technical consensus meeting on headache disorders hosted at WHO headquarters in Geneva in April 2000 [1] and then, crucially, by assimilating the evidence on migraine for WHO's Global Burden of Disease Survey 2000 (GBD2000) (migraine had not featured in the earlier GBD1990). The outcome was conclusive: migraine, on its own, was shown to be amongst the top 20 causes in the world of years of healthy life lost to disability [2]. Headache disorders came in from the cold.

Initially, the GC was a partnership between WHO, International Headache Society (IHS), European Headache Federation (EHF) and World Headache Alliance (WHA), all of whom were co-signatories to the Memorandum of Understanding. It has moved on since. Lifting The Burden is now a legal entity in its own right, incorporated and registered as a charity in the UK, a marker of considerable success in its formative years. More broadly based now, the GC is better described as a collaboration between WHO, international non-governmental organizations, academic institutions and many willing individuals around the world. Its academic base has moved from Imperial College London to the Norwegian University of Science and Technology (NTNU), where it is better supported; the interests and research priorities of the Department of Clinical Neuroscience at NTNU enthusiastically embrace headache and global public health.

The originally conceived three stages of the GC have been described in detail before [3]. In summary, first is to know the nature, scope and scale of the problem-that is, the burden of headache-everywhere in the world ("knowledge for action"). It is perhaps extraordinary that, in 2003, very little was known of the prevalence or burden 


\section{I may, I might, I must}

If you will tell me why the fen appears impassable, I then will tell you why I think that I can get across it if I try.

Marianne Moore (1887-1972)

of any headache disorder for more than half the people of the world [4]: those living in most of the Western Pacific including China, all of South East Asia including India, all of Eastern Europe including Russia, most of Eastern Mediterranean and most of Africa. Second is to exploit this knowledge, as it is gathered, to persuade governments, health-care providers and the public that, on clear evidence, headache must have higher health-care priority ("awareness for action"). Third, and the ultimate purpose of the GC, is to work with local policy-makers and principal stakeholders to plan and implement health-care services for headache, ensuring these are appropriate to local systems, resources and needs ("action for beneficial change").

Changing the world is a challenging task. Rather than suffer Descartes' paralysis from uncertainty [5], Lifting The Burden adopted the indomitable spirit invoked by American poetess, Marianne Moore (Box 1), and set about the task with an aspirational vision (Box 2). It took the three stages of the task apart into multiple steps, all with achievable objectives that, when reassembled at some time in the future, would lead to that vision. ${ }^{1}$

So what has happened in these three arenas during these 7 years?

Filling the very large gaps in knowledge for action has been the first priority. No standard methodology existed for population-based burden-of-headache studies, so Lifting The Burden developed its own. The model calls for a representative mix of urban and rural population samples, encountered by door-to-door "cold-calling" at randomlyselected households; from each household, one adult, also randomly selected, is interviewed; the structured diagnostic questionnaire, based on ICHD-II, is validated in a pilot study within the population to be surveyed.

Applying this model, studies have been completed in Georgia [6-8] and Moldova [9] and have reached the analysis stage in Russia [10], China [11] and India; others are underway in Zambia and Pakistan, and more are

\footnotetext{
${ }_{1}$ A journey of a thousand miles begins with a single step (attributed to Confucius).
}

\section{The Global Campaign's vision}

Lifting The Burden envisions a future world in which headache disorders are recognized everywhere as real, disabling and deserving of medical care. In this future world, all who need care have access to it without artificial barriers.

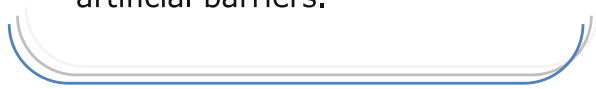

planned in Saudi Arabia, Ethiopia and, possibly, Morocco, Abu Dhabi, Guatemala, Belize, Serbia and Brazil. So far, these have revealed an extraordinarily high prevalence of daily headache in countries of Eastern Europe, highly prevalent migraine in Russia and, especially, in India (as represented by Karnataka State), and a prevalence of migraine in China, where it had been thought to be low, that is not very dissimilar from the global average of $11 \%$ [4].

Lifting The Burden is a partner in Eurolight, a project supported by the European Commission Public Health Executive Agency to survey the impact of headache throughout Europe. This has harvested information from people with headache in Austria, France, Germany, Ireland, Italy, Lithuania, Luxembourg, the Netherlands, Spain and UK [12]. All of this will soon be published.

As for awareness, those present at the International Headache Congress in Kyoto in October 2005 will recall the session presenting the Kyoto Declaration on Headache. This was drafted with the guidance and signed in the presence not only of WHO's Regional Director for the Western Pacific Region but also of representatives of the Japanese Ministry of Health, Labour and Welfare. Lifting The Burden secured the inclusion of headache disorders in the Atlas of Neurological Disorders [13], produced in 2005 jointly by WHO and the World Federation of Neurology (WFN), and as a major chapter in WHO's later publication, Neurological disorders: public health challenges [14]. All of these, not only because they have the imprimatur of WHO but also because their content is compelling, enter the consciousness of politicians, bringing awareness to them of headache as a substantial cause of public ill-health [15]. So, too, does Lifting The Burden's joint review with WHO showing the paucity of headache research in lowand middle-income countries [16], and even more so will the joint global survey for WHO's Atlas of Headache Disorders, due to be published in 2011. The Atlas of 
Headache Disorders, one in the continuing series of Atlases published by WHO, will include data on headache and headache services gathered from more than 100 countries.

Politically more telling than all of these will be the inclusion of migraine and, for the first time, tension-type headache and medication-overuse headache in the new Global Burden of Disease Study 2005 (GBD2005). GBD2005 is a major revision of GBD2000, the importance of which, for the cause of headache, is highlighted above: it is essential for the future that GBD2005 accords due weight to the worldwide burden of headache, and Lifting The Burden has put much into assimilating, analyzing and presenting the evidence on which this depends.

As Lifting The Burden considers models of headache service delivery and organization, and endeavours to make evidence-driven recommendations for change [17], one clear principle is that most headache management belongs in primary care. The numbers of people who need it make this so [17], but it is anyway the case that most headache management does not benefit from involvement of specialists. Non-experts in primary care can do it perfectly well, although they do need some training.

Education is a central pillar of beneficial change [3, 18]. Training doctors to be better at managing headache is a huge undertaking on its own, but completely necessary: the current deficiencies in training, themselves engendered by the low priority given to headache, are at the heart (though not the whole cause) of the universal health-care failures for headache. Education is required at all levels, and therefore an undertaking to be shared-with IHS, EHF and similar organizations, of course, but also with the universities. Within the GC is the Masters Degree in Headache Medicine at Sapienza University, Rome. This annual theoretical and practical course (next year's will be the eighth) is delivered by an international faculty [19]. It is a trainingthe-trainers programme, directed at specialists but with the hope of reaching primary care, the intended target, as the trainees return as trainers to their home countries.

Management by non-experts in primary care can be made better also by the provision of practical clinical management supports, upon which Lifting The Burden embarked by assembling a writing and review group from all world regions in order to ensure multicultural relevance-a cardinal requirement of everything the GC is engaged in. Already produced, or in development, are diagnostic aids applying the criteria of ICHD-II, but simplified; regional management guidelines developed, where these exist, by harmonizing national guidelines [20]; information sheets for patients to aid understanding and promote compliance with treatment [21]; and universally acceptable indices of impact and treatment outcome [22]. The last was developed at a technical consensus meeting on headache outcome measures at WHO headquarters in April 2006, and follow-up validation and evaluation studies are being conducted in six countries.

The Handbook of Headache, written by authors from all over the world and to be published next year in print and electronically, is also aimed at non-experts. It will be a supplement to these aids, providing detail when this is required.

Because good translation is crucial to multicultural relevance, Lifting The Burden has developed translation standards and protocols for GC materials [23].

Lifting The Burden is working with, and supporting, the Cochrane Collaboration, fostering systematic reviews of treatments for headache. One of the purposes is to be able to advise $\mathrm{WHO}$ on revisions to their essential medicines list which, in time, will encourage availability worldwide of the drugs most needed to treat headache effectively.

As for actual intervention, Lifting The Burden has developed a headache-service model, to be tested soon in Georgia and later, if plans go forward, in Serbia, Bulgaria and Abu Dhabi. The model is adaptable, but involves first assessing local need, together with willingness to pay, upon which sustainability will depend. The next steps in Georgia are to establish three clinics, provide free care and drugs to geographically-defined populations and show the benefits of treatment to people and of the service to population health. Only once these benefits are apparent, the service will charge according to willingness to pay in order to become self-sustaining.

Ultimately, Lifting The Burden must evaluate what it helps to create, and amend it, in an iterative process if necessary, to achieve what is best possible. This raises a fundamental question: what is a good headache service? Surprisingly, or perhaps not, "quality" in the context of headache services has no accepted definition. Indeed it is not easily defined, although in part it must lie in the attainment of good outcomes, which can be measured. In preparing its proposals for headache-service quality evaluation, soon to be published, Lifting The Burden has undertaken a worldwide consultation.

This is a summary of what has happened. Not everything has been included. We believe Lifting The Burden can be pleased with and proud of these first 7 years. The activities represent many more than a single step (see footnote 1); more importantly, the steps are all in one and the right direction - each part of a cohesive, managed project directed towards a clear purpose. They involve actions in 28 countries, a seventh of the world's total. The collaborations underpinning them include WHO of course, its headquarters in Geneva and the Regional Offices for South East Asia and Western Pacific; they include IHS and, notably, its Russian Linguistic Subcommittee, EHF, WHA, WFN and the Pain, Palliative and Supportive Care (PaPaS) 
group of the Cochrane Collaboration. At national level they include: in Austria: Konventhospital Barmherzige Brüder, Linz; in Belgium: University of Ghent; in Brazil: the Federal University of Santa Catarina, Florianopolis, and Botucatu Medical School; in China: the Ministry of Health, the PLA General Hospital, Beijing, the Fourth Military Medical University, Xian, Xiaya Hospital of Centre-south University, Changsha, Affiliated Huashan Hospital of Fudan University, Shanghai, the First Affiliated Hospital of Sun Yat-sen University, Guangzhou and the First Hospital of Jilin University, Changchun; in Denmark: the Danish Headache Centre, Glostrup, and the University of Copenhagen; in Ethiopia: the University of Addis Ababa; in France: Hôpital Pasteur, Nice, and Hôpital Lariboisière, Paris; in Georgia: Tbilisi Medical University; in Germany: the University of Essen and the Institute for Health and Rehabilitation Sciences, Ludwig Maximilians University, Munich; in India: the National Institute for Mental Health and Neurosciences, Bangalore; in Italy: the National Neurological Institute C Mondino, Pavia, Sapienza University, Rome, Department of Neurology Policlinic of Monza, the University of Turin and the Neurological Institute Carlo Besta, Milan; in Japan: the Ministry of Health, Labour and Welfare and the International Headache Center, Kawasaki, Kanagawa; in Luxembourg: Centre de Recherche Public de la Santé; in Moldova: Chisinau State Medical and Pharmaceutical University; in the Netherlands: Medisch Centrum Boerhaave, Amsterdam; in Norway: the Norwegian National Headache Centre and the Norwegian University of Science and Technology, Trondheim; in Pakistan: the University of Karachi; in Portugal: Hospital da Luz, Lisbon; in Russia: Setchenov Moscow Medical Academy and the Institute of Sociology, Russian Academy of Sciences, Moscow; in Saudi Arabia: King Abdullah International Medical Research Center, Riyadh, and the Saudi Arabia National Guard; in Serbia: the Ministry of Health and the Institute of Neurology and School of Medicine, Belgrade; in Spain: University Clinic Hospital, Valencia University; in Sri Lanka: the University of Colombo; in United Arab Emirates: the Health Authority_Abu Dhabi (HAAD); in UK: Imperial College London, the City of London Migraine Clinic, the University of Oxford and Isis Medical Media Ltd, Tonbridge; in USA: Albert Einstein College of Medicine, Bronx NY, Brigham and Women's Hospital, Boston MA, Duke University, Durham NC, Geisinger Clinic, Center for Health Research, Danville, PA, Michigan State University, East Lansing MI, the New England Center for Headache, Stamford CT, Park Nicollet Headache Clinic \& Research Center, Minneapolis MN, Roosevelt Hospital, New York NY, Uniformed Services University, Bethesda MD and the University of North Carolina, Chapel Hill NC; in Zambia: Chainama Hills College Hospital, Lusaka. And at individual level, they include many many people, far too numerous to list.

All of these, and the many sponsors, we warmly thank.

Conflicts of interest The authors are the directors and trustees of Lifting The Burden. TJS is honorary Global Campaign Director.

Open Access This article is distributed under the terms of the Creative Commons Attribution Noncommercial License which permits any noncommercial use, distribution, and reproduction in any medium, provided the original author(s) and source are credited.

\section{References}

1. World Health Organization (2000) Headache disorders and public health: education and management implications. WHO, Geneva

2. World Health Organization (2001) World health report 2001. WHO, Geneva

3. Steiner TJ (2004) Lifting the burden: the global campaign against headache. Lancet Neurol 3:204-205

4. Stovner LJ, Hagen K, Jensen R, Katsarava Z, Lipton RB, Scher AI, Steiner TJ, Zwart J-A (2007) The global burden of headache: a documentation of headache prevalence and disability worldwide. Cephalalgia 27:193-210

5. Descartes R (1901, reprinted 1988) Principles of human knowledge. The meditations and selections from the principles (trans: Veitch J). Open Court, IL, p 130

6. Kukava M, Dzagnidze A, Mirvelashvili E, Djibuti M, Fritsche G, Jensen R, Stovner LJ, Steiner TJ, Katsarava Z (2007) Validation of a Georgian language headache questionnaire in a populationbased sample. J Headache Pain 8:321-324

7. Katsarava Z, Dzagnidze A, Kukava M, Mirvelashvili E, Djibuti M, Janelidze M, Jensen R, Stovner LJ, Steiner TJ (2009) Prevalence of cluster headache in the Republic of Georgia: results of a population-based study and methodological considerations. Cephalalgia 29:949-952

8. Katsarava Z, Dzagnidze A, Kukava M, Mirvelashvili E, Djibuti M, Janelidze M, Jensen R, Stovner LJ, Steiner TJ (2009) Primary headache disorders in the Republic of Georgia: prevalence and risk factors. Neurology 73:1796-1803

9. Moldovanu I, Pavlic G, Odobescu S, Rotaru L, Craciun C, Ciobanu L, Corcea G, Steiner T, Katsarava Z (2007) The prevalence of headache disorders in the Republic of Moldova: a populationbased study. Cephalalgia 27:673

10. Ayzenberg I, Katsarava Z, Mathalikov R, Chernysh M, Osipova V, Tabeeva G, Steiner TJ, on behalf of Lifting The Burden: the Global Campaign to Reduce Burden of Headache Worldwide and the Russian Linguistic Subcommittee of the International Headache Society (2010) The burden of headache in Russia: validation of the diagnostic questionnaire in a population-based sample. Eur $\mathrm{J}$ Neurol (Epub ahead of print)

11. Yu S-Y, Cao X-T, Zhao G, Yang X-S, Qiao X-Y, Fang Y-N, Feng J-C, Liu R-Z, Steiner TJ (2010) The burden of headache in China: validation of diagnostic questionnaire for a populationbased survey. J Headache Pain (accepted)

12. Andrée C, Vaillant M, Barre J, Katsarava Z, Lainez JM, Lair M-L, Lanteri-Minet M, Lampl C, Steiner TJ, Stovner LJ, Tassorelli C, Sándor PS (2010) Development and validation of the EUROLIGHT questionnaire to evaluate the burden of primary headache disorders in Europe. Cephalalgia 30:1082-1100

13. World Health Organization. World Federation of Neurology (2004) Atlas: country resources for neurological disorders. WHO, Geneva 
14. World Health Organization (2007) Neurological disorders: public health challenges. WHO, Geneva

15. Martelletti P, Steiner TJ, Bertolote JM, Dua T, Saraceno B (2007) The definitive position of headache among the major public health challenges: an end to the slippery slope of disregard (editorial). J Headache Pain 8:149-151

16. Mateen F, Dua T, Steiner T, Saxena S (2008) Headache disorders in developing countries: research over the past decade. Cephalalgia 28:1107-1114

17. Antonaci F, Valade D, Lanteri-Minet M, Láinez JM, Jensen J, Steiner TJ (2008) Proposals for the organisation of headache services in Europe. Intern Emerg Med 3:S25-S28

18. Steiner TJ (2005) Lifting The Burden: the global campaign to reduce the burden of headache worldwide. J Headache Pain 6:373-377
19. Martelletti P, Haimanot RT, Lainez MJA, Rapoport AM, Ravishankar K, Sakai F, Silberstein SD, Vincent M, Steiner TJ (2005) The Global Campaign to Reduce the Burden of Headache Worldwide. The International Team for Specialist Education (ITSE). J Headache Pain 6:261-263

20. Steiner TJ, Paemeleire K, Jensen R, Valade D, Savi L, Lainez MJA, Diener H-C, Martelletti P, Couturier EGM (2007) European principles of management of common headache disorders in primary care. J Headache Pain 8(suppl 1):S3-S21

21. Steiner TJ (2007) Information for patients. J Headache Pain 8(Suppl 1):S26-S39

22. Steiner TJ (2007) The HALT and HART indices. J Headache Pain 8(Suppl 1):S22-S25

23. Peters M (2007) Translation protocols. J Headache Pain 8(Suppl 1):S40-S47 